\title{
A DECOMPOSITION OF EQUIVARIANT K-THEORY IN TWISTED EQUIVARIANT K-THEORIES
}

\author{
JOSÉ MANUEL GÓMEZ AND BERNARDO URIBE
}

\begin{abstract}
For $G$ a finite group and $X$ a $G$-space on which a normal subgroup $A$ acts trivially, we show that the $G$-equivariant K-theory of $X$ decomposes as a direct sum of twisted equivariant $\mathrm{K}$-theories of $X$ parametrized by the orbits of the conjugation action of $G$ on the irreducible representations of $A$. The twists are group 2-cocycles which encode the obstruction of lifting an irreducible representation of $A$ to the subgroup of $G$ which fixes the isomorphism class of the irreducible representation.
\end{abstract}

\section{INTRODUCTION}

Suppose that we have a group extension of finite groups

$$
1 \rightarrow A \stackrel{\iota}{\rightarrow} G \stackrel{\pi}{\rightarrow} Q \rightarrow 1
$$

The purpose of this article is to study the $G$-equivariant K-theory $K_{G}^{*}(X)$, on compact, Hausdorff $G$-spaces $X$ for which the action of $A$ is trivial.

Equivariant K-theory is an equivariant generalized cohomology theory that is constructed out of $G$-equivariant vector bundles. Its basic properties were derived in $[10$.

Whenever $X$ is a $G$-space such that $A$ acts trivially and $p: E \rightarrow X$ is a $G$ equivariant vector bundle, then we can regard $E$ as an $A$-equivariant vector bundle and thus the fibers of $E$ can be seen as $A$-representations. Decomposing $E$ into $A$-isotypical pieces (see [10, Proposition 2.2]), we obtain a decomposition of $E$ as an $A$-equivariant vector bundle

$$
\bigoplus_{[\tau] \in \operatorname{Irr}(A)} \mathbb{V}_{\tau} \otimes \operatorname{Hom}_{A}\left(\mathbb{V}_{\tau}, E\right) \cong E
$$

Here $\mathbb{V}_{\tau}$ denotes the $A$-vector bundle $\pi_{1}: X \times V_{\tau} \rightarrow X$ associated to an irreducible representation $\tau: A \rightarrow U\left(V_{\tau}\right)$ and $\operatorname{Irr}(A)$ denotes the set of isomorphism classes of complex irreducible $A$-representations.

It is important to point out that this decomposition is one of $A$-vector bundles and not one of $G$-vector bundles since in general the bundles $\mathbb{V}_{\tau} \otimes \operatorname{Hom}_{A}\left(\mathbb{V}_{\tau}, E\right)$ do not possess the structure of a $G$-vector bundle. The key observation of this work is that the sum $\bigoplus_{[\tau] \in \operatorname{Irr}(A)} \mathbb{V}_{\tau} \otimes \operatorname{Hom}_{A}\left(\mathbb{V}_{\tau}, E\right)$ can be rearranged using the

2010 Mathematics Subject Classification. 19L47, 19L50.

Key words and phrases. Equivariant K-theory, twsited K-theory, twisted equivariant K-theory.

Both authors acknowledge and thank the financial support provided by the Max Planck Institute for Mathematics and by COLCIENCIAS through grant number FP44842-617-2014 of the Fondo Nacional de Financiamiento para la Ciencia, la Tecnología y la Innovación. The second author acknowledges and thanks the financial support provided by the Alexander Von Humboldt Foundation. 
different orbits of the action of $Q$ on $\operatorname{Irr}(A)$ as to obtain a decomposition of $E$ in terms of $G$-vector bundles. Moreover, we show that the factors obtained in this refined decomposition naturally define vector bundles that are used to defined twisted forms of equivariant K-theory and in this way we obtain a decomposition of $K_{G}^{*}(X)$ as a direct sum of twisted forms of equivariant K-theory. To make this precise suppose that $\tau: A \rightarrow U\left(V_{\tau}\right)$ is an irreducible $A$-representation. Let $G_{[\tau]}$ (resp. $Q_{[\tau]}$ ) denote the isotropy group of the action of $G$ (resp. $Q$ ) at $[\tau] \in \operatorname{Irr}(A)$. These groups fit into a group extension of the form

$$
1 \rightarrow A \stackrel{\iota}{\rightarrow} G_{[\tau]} \stackrel{\pi}{\rightarrow} Q_{[\tau]} \rightarrow 1
$$

thus defining a 2-cocycle $\alpha_{\tau} \in Z^{2}\left(Q_{[\tau]}, \mathbb{S}^{1}\right)$ which is the data needed to define the $\alpha_{\tau}$-twisted $Q_{[\tau]}$-equivariant K-theory groups ${ }^{\alpha_{\tau}} K_{Q_{[\tau]}}^{*}(X)$ (see [2, Section 7$]$ for the definition). With this notation we can state the following theorem which is the main result of this article.

Theorem 3.4. Suppose that $A \subset G$ is a normal subgroup and $X$ is a compact, Hausdorff $G$-space on which $A$ acts trivially. Then there is a natural isomorphism

$$
\Psi_{X}: K_{G}^{*}(X) \stackrel{\cong}{\rightarrow} \bigoplus_{[\tau] \in G \backslash \operatorname{Irr}(A)} \alpha_{\tau} K_{Q_{[\tau]}}^{*}(X)
$$

where $[\tau]$ runs over the orbits of $G$ on $\operatorname{Irr}(A)$, This isomorphism is functorial on maps $X \rightarrow Y$ of $G$-spaces on which $A$ acts trivially.

We remark that the previous theorem also holds in the case of $G$ being a compact Lie group. However we chose to work first with finite groups because in this case we can obtain explicit formulas for the cocycles used to twist equivariant K-theory. The general case will be handled in a sequel to this article.

The layout of this article is as follows. In Section 1 we study the problem of extending homomorphisms of finite groups. In particular, the cocycles $\alpha_{\tau} \in$ $Z^{2}\left(Q_{[\tau]}, \mathbb{S}^{1}\right)$ that appear in Theorem 3.4 are constructed in this section. In Section 2 we construct a twisted form of equivariant K-theory using vector bundles that come equipped with a prescribed fiberwise representation. In Section 3 we prove Theorem 3.4 which is the main result of this work. In Section 4 we relate Theorem 3.4 to the Atiyah-Segal completion theorem. In Section 5 we provide a formula for the third differential of the Atiyah-Hirzebruch spectral sequence that computes $K_{G}^{*}(X)$ whenever $A$ acts trivially on $X$. Finally, in Section 6 some explicit computations are provided for the dihedral group $D_{8}$.

Throughout this work all the spaces in sight will be compact and Hausdorff endowed with a continuous action of the finite group $G$ unless stated otherwise.

\section{EXTENSIONS OF HOMOMORPHISMS OF FINITE GROUPS}

In this section we study extensions of homomorphisms of finite groups. Our main goal is to show that the obstructions for finding such extensions can be studied using group cohomology. We remark that the material in this section may be known to experts but we include the main ingredients that will be used throughout this article for completeness. We refer the reader to [1, Chapter I] and [7] for background on group cohomology. 
Consider the group extension of finite groups

$$
1 \rightarrow A \stackrel{\iota}{\rightarrow} G \stackrel{\pi}{\rightarrow} Q \rightarrow 1
$$

and fix a given homomorphism of groups $\rho: A \rightarrow U$. We want to study the conditions under which the homomorphism $\rho$ may be extended to a homomorphism $\widetilde{\rho}: G \rightarrow U$ in such a way that $\widetilde{\rho} \circ \iota=\left.\widetilde{\rho}\right|_{A}=\rho$.

First note that since $A$ is normal in $G$, the group $G$ acts on the left on the set $\operatorname{Hom}(A, U)$ of homomorphisms from $A$ to $U$ : for a homomorphism $\chi: A \rightarrow U$ and $g \in G$ we define the homomorphism $g \cdot \chi$ by the equation

$$
(g \cdot \chi)(a):=\chi\left(g^{-1} a g\right) .
$$

Second note that the group $U$ acts on the right on $\operatorname{Hom}(A, U)$ by conjugation: for a homomorphism $\chi: A \rightarrow U$ and $M \in U$ we define the homomorphism $\chi \cdot M$ by the equation

$$
(\chi \cdot M)(a):=M^{-1} \chi(a) M .
$$

Further note that this left $G$ action on $\operatorname{Hom}(A, U)$ commutes with the right $U$ action.

If $\rho$ were to be extended to $\widetilde{\rho}: G \rightarrow U$ then we would have the equality

$$
(g \cdot \rho)(a)=\rho\left(g^{-1} a g\right)=\widetilde{\rho}(g)^{-1} \rho(a) \widetilde{\rho}(g)
$$

thus implying that the homomorphisms $g \cdot \rho$ and $\rho$ are conjugate to each other by an element $\widetilde{\rho}(g)$ in $U$, or in other words that $g \cdot \rho=\rho \cdot \widetilde{\rho}(g)$. In particular this implies that there must exist a homomorphism $f: G \rightarrow \operatorname{Inn}(U)$ from $G$ to the inner automorphisms of $U$ such that we have the equation

$$
g \cdot \rho=f(g) \circ \rho
$$

and that $\rho \in[\operatorname{Hom}(A, U) / U]^{G}$, i.e. the class of $\rho$ is $G$-invariant on the set equivalence classes of homomorphisms up to conjugation.

Therefore the first obstruction for the existence of the extension $\widetilde{\rho}$ of $\rho$ is the existence of a homomorphism $f: G \rightarrow \operatorname{Inn}(U)$ such that the following diagram commutes

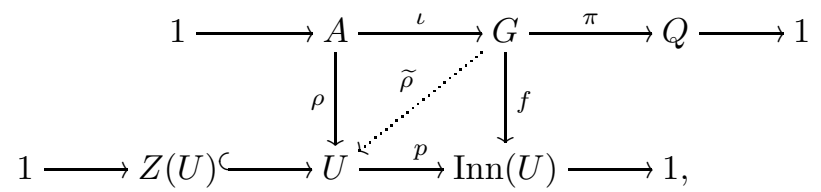

where the homomorphism $p$ is the canonical homomorphism, and $Z(U)$ is the center of $U$, and that the class of $\rho$ up to $U$ conjugation is $G$-invariant, i.e $\rho \in$ $[\operatorname{Hom}(A, U) / U]^{G}$.

1.1. Extension of homomorphisms. Let us suppose that we are in the situation described in diagram (1.1) with $\rho \in[\operatorname{Hom}(A, U) / U]^{G}$. In what follows we will show that the obstruction for the existence of the extension $\widetilde{\rho}: G \rightarrow U$ lies in $H^{2}(Q, Z(U))$.

Take a set theoretical section $\sigma: Q \rightarrow G$ of the homomorphism $\pi: G \rightarrow Q$ satisfying $\pi(\sigma(q))=q$ for all $q \in Q$. Choose $\sigma$ such that $\sigma(1)=1$. Define $\chi: Q \times Q \rightarrow A$ by the equation

$$
\chi\left(q_{1}, q_{2}\right)=\sigma\left(q_{1} q_{2}\right)^{-1} \sigma\left(q_{1}\right) \sigma\left(q_{2}\right)
$$


and notice that $\chi\left(q_{1}, q_{2}\right)$ belongs to $A$ since $\pi\left(\chi\left(q_{1}, q_{2}\right)\right)=1$ and moreover that $\chi$ is normalized in the sense that $\chi\left(q_{1}, q_{2}\right)=1$ whenever $q_{1}=1$ or $q_{2}=1$. Define also the set theoretical map $\psi: Q \rightarrow \operatorname{Aut}(A)$ by the equation

$$
\psi(q)(a)=\sigma(q)^{-1} a \sigma(q) .
$$

The pair $(\chi, \psi)$ defines a nonabelian group 2-cocycle with automorphisms and it satisfies the cocycle equation

$$
\chi\left(q_{1} q_{2}, q_{3}\right) \psi\left(q_{3}\right)\left(\chi\left(q_{1}, q_{2}\right)\right)=\chi\left(q_{1}, q_{2} q_{3}\right) \chi\left(q_{2}, q_{3}\right) .
$$

Using the 2 -cocycle $(\chi, \psi)$ we can endow the set $Q \times A$ with a group structure as follows

$$
\left(q_{1}, a_{1}\right)\left(q_{2}, a_{2}\right)=\left(q_{1} q_{2}, \chi\left(q_{1}, q_{2}\right) \psi\left(q_{2}\right)\left(a_{1}\right) a_{2}\right) .
$$

Denote this group by $Q \ltimes_{(\chi, \psi)} A$ and note that the maps

$$
\begin{array}{rlrl}
G & \rightarrow Q \ltimes_{(\chi, \psi)} A & Q \ltimes_{(\chi, \psi)} A & \rightarrow G \\
g & \mapsto\left(\pi(g), \sigma(\pi(g))^{-1} g\right) & (q, a) & \mapsto \sigma(q) a
\end{array}
$$

become isomorphisms of groups one inverse to the other; therefore we may identify the group $G$ with the group $Q \ltimes_{(\chi, \psi)} A$.

On the other hand, let us consider the inner automorphisms of $U$ defined by the elements $f(\sigma(q))$ and choose elements $M_{q} \in U$ such that

$$
p\left(M_{q}\right)=f(\sigma(q))
$$

for all $q \in Q$; choose $M_{1}=1$. In particular we have that

$$
\rho\left(\sigma(q)^{-1} a \sigma(q)\right)=M_{q}^{-1} \rho(a) M_{q}
$$

for all $q \in Q$ and $a \in A$.

For $q_{1}, q_{2}$ in $Q$ we notice that

$$
p\left(\rho\left(\chi\left(q_{1}, q_{2}\right)\right) M_{q_{2}}^{-1} M_{q_{1}}^{-1} M_{q_{1} q_{2}}\right)=1
$$

and thus $\rho\left(\chi\left(q_{1}, q_{2}\right)\right) M_{q_{2}}^{-1} M_{q_{1}}^{-1} M_{q_{1} q_{2}}$ belongs to the center $Z(U)$.

Definition 1.4. Consider the commutative diagram of groups of (1.1) with $\rho \in$ $[\operatorname{Hom}(A, U) / U]^{G}$, the set theoretical section $\sigma: Q \rightarrow G$ and the lifts $M_{q} \in U$ of the elements $f(\sigma(q))$ with $M_{1}=1$. Define the map $\alpha_{\rho}: Q \times Q \rightarrow Z(U)$ by the equation

$$
\alpha_{\rho}\left(q_{1}, q_{2}\right):=\rho\left(\chi\left(q_{1}, q_{2}\right)\right) M_{q_{2}}^{-1} M_{q_{1}}^{-1} M_{q_{1} q_{2}} .
$$

The following lemma shows that associated to $\alpha_{\rho}$ we have a cohomology class that does not depend on the choices made above. The proof follows by direct computations and is left as an exercise to the reader.

Lemma 1.5. The map $\alpha_{\rho}: Q \times Q \rightarrow Z(U)$ satisfies the 2-cocycle condition, i.e. for every $q_{1}, q_{2}, q_{3} \in Q$ the equality

$$
\alpha_{\rho}\left(q_{1}, q_{2} q_{3}\right) \alpha_{\rho}\left(q_{2}, q_{3}\right)=\alpha_{\rho}\left(q_{1} q_{2}, q_{3}\right) \alpha_{\rho}\left(q_{1}, q_{2}\right)
$$

is satisfied, thus making $\alpha_{\rho}$ a 2-cocycle of the group $Q$ with coefficients in the abelian group $Z(U)$ seen as a trivial $Q$-module. Moreover, the cohomology class $\left[\alpha_{\rho}\right] \in H^{2}(Q, Z(U))$ is well defined. Namely, it does not depend on the choice of section $\sigma$ nor on the choice of lifts $M_{q}$ 's. 
Proposition 1.6. The obstruction for the existence of the extension $\widetilde{\rho}: G \rightarrow U$ of $\rho: A \rightarrow U$ fitting into diagram (1.1) and satisfying $\rho \in[\operatorname{Hom}(A, U) / U]^{G}$ is the cohomology class $\left[\alpha_{\rho}\right] \in H^{2}(Q, Z(U))$. Namely, $\widetilde{\rho}$ exists if and only if $\left[\alpha_{\rho}\right]=1$.

Proof. Let us suppose there is a group homomorphism $\widetilde{\rho}: G \rightarrow U$ extending $\rho$ : $A \rightarrow U$ and fitting into the diagram (1.1). Take any section $\sigma: Q \rightarrow G$ with $\sigma(1)=1$ and choose $M_{q}:=\widetilde{\rho}(\sigma(q))$. Then

$$
\begin{aligned}
\alpha_{\rho}\left(q_{1}, q_{2}\right) & =\rho\left(\chi\left(q_{1}, q_{2}\right)\right) M_{q_{2}}^{-1} M_{q_{1}}^{-1} M_{q_{1} q_{2}} \\
& =\widetilde{\rho}\left(\sigma\left(q_{1} q_{2}\right)^{-1} \sigma\left(q_{1}\right) \sigma\left(q_{2}\right)\right) \widetilde{\rho}\left(\sigma\left(q_{2}\right)\right)^{-1} \widetilde{\rho}\left(\sigma\left(q_{1}\right)\right)^{-1} \widetilde{\rho}\left(\sigma\left(q_{1} q_{2}\right)\right)=1
\end{aligned}
$$

and therefore $\left[\alpha_{\rho}\right]=1$.

Let us now suppose that there exist $\varepsilon: Q \rightarrow Z(U)$ such that $\delta \varepsilon=\alpha_{\rho}$; this implies that for $q_{1}, q_{2} \in Q$ we obtain

$$
\varepsilon\left(q_{2}\right) \varepsilon\left(q_{1} q_{2}\right)^{-1} \varepsilon\left(q_{1}\right)=\rho\left(\chi\left(q_{1}, q_{2}\right)\right) M_{q_{2}}^{-1} M_{q_{1}}^{-1} M_{q_{1} q_{2}} .
$$

Since the image of $\varepsilon$ lies in the center of $U$, we may define $\widetilde{M}_{q}:=M_{q} \varepsilon(q)$ thus obtaining the equation $\widetilde{M}_{q_{1}} \widetilde{M}_{q_{2}}=\widetilde{M}_{q_{1} q_{2}} \rho\left(\chi\left(q_{1}, q_{2}\right)\right)$. Consider the map

$$
\Psi: Q \ltimes_{(\chi, \psi)} A \rightarrow U, \quad(q, a) \mapsto \widetilde{M}_{q} \rho(a) .
$$

It is straight forward to verify that $\Psi$ is a group homomorphism. Composing the map $\Psi$ with the isomorphism $G \rightarrow Q \ltimes_{(\chi, \psi)} A, g \mapsto(\pi(g), \sigma(\pi(g)))^{-1} g$ we may define the homomorphism

$$
\widetilde{\rho}: G \rightarrow U, \quad g \mapsto \widetilde{M}_{\pi(g)} \rho\left(\sigma(\pi(g))^{-1} g\right) .
$$

Since $\widetilde{\rho}(a)=\rho\left(\sigma(\pi(a))^{-1} a\right)=\rho(a)$ for $a \in A$ we obtain the desired homomorphism $\tilde{\rho}: G \rightarrow U$ fitting into diagram (1.1).

Remark 1.7. Note that in the case that $U$ is abelian we may find the obstruction for the existence of the extension $\widetilde{\rho}: G \rightarrow U$ from the Lyndon-Hochschild-Serre spectral sequence associated to the group extension $1 \rightarrow A \rightarrow G \rightarrow Q \rightarrow 1$. The LHS spectral sequence converges to $H^{*}(G, U)$ and its second page is $E_{2}^{p, q} \cong$ $H^{p}\left(Q, H^{q}(A, U)\right)$. Since $E_{1}^{0,1} \cong H^{1}(A, U)=\operatorname{Hom}(A, U)$, a homomorphism $\rho \in$ $H^{1}(A, U)$ extends to one in $H^{1}(G, U)=\operatorname{Hom}(G, U)$ if $\rho$ survives all pages of the spectral sequence. The first obstruction for the extension is $d_{1} \rho$, and we have that $d_{1} \rho=0$ if and only if $\rho$ is $G$-invariant, i.e. $\rho \in \operatorname{Hom}(A, U)^{G}$. If $\rho$ is $G$-invariant, the second and last obstruction for the extension is $d_{2} \rho \in E_{2}^{2,0} \cong H^{2}(Q, U)$, which is precisely the cohomology class $\left[\alpha_{\rho}\right]$ of Proposition 1.6. In this case the extension exists if and only if $\rho$ is $G$ invariant and $\left[\alpha_{\rho}\right]=0$.

1.2. Extensions of irreducible representations. Consider now the case of an irreducible representation $\rho: A \rightarrow U\left(V_{\rho}\right)$ where $V_{\rho}$ is a complex representation of $A$ and $U\left(V_{\rho}\right)$ denotes the group of unitary transformations of $V_{\rho}$. To start we have the following lemma whose proof is left to the reader.

Lemma 1.8. Suppose that for all $g \in G$ the irreducible representation $g \cdot \rho$ is isomorphic to $\rho$, or what is the same that $\rho \in[\operatorname{Hom}(A, U) / U]^{G}$. Then there exist a unique homomorphism $f: G \rightarrow \operatorname{Inn}\left(U\left(V_{\rho}\right)\right)$ making the following diagram 
commutative

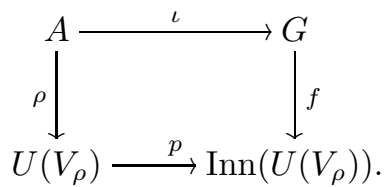

Proposition 1.9. Consider the group extension $1 \rightarrow A \rightarrow G \rightarrow Q \rightarrow 1$ of finite groups and $\rho: A \rightarrow U\left(V_{\rho}\right)$ an irreducible representation of $A$ such that $(g \cdot \rho) \cong$ $\rho$ for all $g \in G$. Then the representation $\rho$ may be extended to an irreducible representation $\widetilde{\rho}: G \rightarrow U\left(V_{\rho}\right)$ if and only if $\left[\alpha_{\rho}\right]$ is trivial in $H^{2}\left(Q, \mathbb{S}^{1}\right)$.

Proof. By Lemma 1.8 we know that there exists $f: G \rightarrow \operatorname{Inn}\left(U\left(V_{\rho}\right)\right)$ making diagram (1.1) commutative. By Proposition 1.6 we know that the obstruction for the existence of the extension is $\left[\alpha_{\rho}\right] \in H^{2}\left(Q, Z\left(U\left(V_{\rho}\right)\right)\right)$, and since the center of $U\left(V_{\rho}\right)$ is isomorphic to $\mathbb{S}^{1}$, the result follows.

\section{TWISTED EQUiVARIANT K-THEORY VIA REPRESENTATIONS}

In this section we provide a description of a twisted form of equivariant K-theory via vector bundles that come equipped with a prescribed fiberwise representation of a finite group. To start suppose that $A$ is a finite group. Assume that $A$ is a normal subgroup of a finite group $G$ so that we have a group extension

$$
1 \rightarrow A \stackrel{\iota}{\rightarrow} G \stackrel{\pi}{\rightarrow} Q \rightarrow 1
$$

with $Q=G / A$. Let $G$ act on a compact and Hausdorff space $X$ in such a way that for every $x \in X$ we have $A \subset G_{x}$. In other words, the subgroup $A$ acts trivially on $X$. Let $p: E \rightarrow X$ be a $G$-equivariant vector bundle. We can give $E$ a Hermitian metric that is invariant under the action of $A$. If we see $p: E \rightarrow X$ as an $A$-vector bundle then as the action of $A$ on $X$ is trivial by [10. Proposition 2.2] we have a natural isomorphism of $A$-vector bundles

$$
\begin{aligned}
\bigoplus_{[\tau] \in \operatorname{Irr}(A)} \mathbb{V}_{\tau} \otimes \operatorname{Hom}_{A}\left(\mathbb{V}_{\tau}, E\right) & \rightarrow E \\
v \otimes f & \mapsto f(v) .
\end{aligned}
$$

In the above equation $\operatorname{Irr}(A)$ denotes the set of isomorphism classes of complex irreducible $A$-representations and if $\tau: A \rightarrow U\left(V_{\tau}\right)$ is an $A$-representation then $\mathbb{V}_{\tau}$ denotes the $A$-vector bundle $\pi_{1}: X \times V_{\tau} \rightarrow X$. It is important to point out that the decomposition given in (2.1) is a decomposition as $A$-equivariant vector bundles and not as $G$-equivariant vector bundles since in general the terms on the left hand side of (2.1) do not have the structure of a $G$-equivariant vector bundle. With this in mind we have the following definition.

Definition 2.2. Suppose that $\rho: A \rightarrow U\left(V_{\rho}\right)$ is a complex irreducible representation and that $1 \rightarrow A \rightarrow G \rightarrow Q \rightarrow 1$ is a group extension of finite groups. A $(G, \rho)$-equivariant vector bundle over $X$ is a $G$-vector bundle $p: E \rightarrow X$ such that the map

$$
\begin{aligned}
\mathbb{V}_{\rho} \otimes \operatorname{Hom}_{A}\left(\mathbb{V}_{\rho}, E\right) & \rightarrow E \\
v \otimes f & \mapsto f(v)
\end{aligned}
$$

is an isomorphism of $A$-vector bundles. 
In other words, a $(G, \rho)$-equivariant vector bundle is a $G$-equivariant vector bundle $p: E \rightarrow X$ that satisfies the following property: for every $x \in X$ the $A$ representation $E_{x}$ is isomorphic to a direct sum of the representation $\rho$; that is, the only irreducible $A$-representation that appears in the fibers of $E$ is $\rho$. We can define a direct summand of the equivariant K-theory using $(G, \rho)$-equivariant vector bundles. For this let $\operatorname{Vec}_{G, \rho}(X)$ denote the set of isomorphism classes of $(G, \rho)$-equivariant vector bundles, where two $(G, \rho)$-equivariant vector bundles are isomorphic if they are isomorphic as $G$-vector bundles. Notice that if $E_{1}$ and $E_{2}$ are two $(G ; \rho)$-equivariant vector bundles then so is $E_{1} \oplus E_{2}$. Therefore $\operatorname{Vec}_{G, \rho}(X)$ is a semigroup.

Definition 2.3. Assume that $G$ acts on a compact space $X$ in such a way that $A$ acts trivially on $X$. We define $K_{G, \rho}^{0}(X)$, the $(G, \rho)$-equivariant K-theory of $X$, as the Grothendieck construction applied to $\operatorname{Vec}_{G, \rho}(X)$. For $n>0$ the group $K_{G, \rho}^{n}(X)$ is defined as $\widetilde{K}_{G, \rho}^{0}\left(\Sigma^{n} X_{+}\right)$, where as usual $X_{+}$denotes the space $X$ with an added base point.

The goal of this section is to provide a description of the previous equivariant $\mathrm{K}$-groups in terms of the usual twisted equivariant $\mathrm{K}$-groups as defined for example in [2, Section 7].

Suppose that $\rho: A \rightarrow U\left(V_{\rho}\right)$ is a complex irreducible representation with the property that $g \cdot \rho$ is isomorphic to $\rho$ for every $g \in G$. Fix an assignment $\sigma: Q \rightarrow G$ such that $\pi(\sigma(q))=q$ for all $q \in Q$ with $\sigma(1)=1$. For each $q \in Q$ fix an element $M_{q} \in U\left(V_{\rho}\right)$ such that

$$
\rho\left(\sigma(q)^{-1} a \sigma(q)\right)=M_{q}^{-1} \rho(a) M_{q}
$$

for all $a \in A$. We choose $M_{1}=1$. Let $\alpha_{\rho} \in Z^{2}\left(Q, \mathbb{S}^{1}\right)$ be the cocycle corresponding to $\rho$ constructed in Proposition [1.9. Using the cocycle $\alpha_{\rho} \in Z^{2}\left(Q, \mathbb{S}^{1}\right)$ we can construct a central extension of $Q$ by $\mathbb{S}^{1}$ in the following way: as a set define

$$
\widetilde{Q}_{\alpha_{\rho}}=\left\{(q, z) \mid q \in Q, z \in \mathbb{S}^{1}\right\}
$$

and the product structure in $\widetilde{Q}_{\alpha_{\rho}}$ is given by the assignment

$$
\left(q_{1}, z_{1}\right)\left(q_{2}, z_{2}\right):=\left(q_{1} q_{2}, \alpha_{\rho}\left(q_{1}, q_{2}\right) z_{1} z_{2}\right)
$$

The group $\widetilde{Q}_{\alpha_{\rho}}$ is a compact Lie group that fits into a central extension

$$
1 \rightarrow \mathbb{S}^{1} \rightarrow \widetilde{Q}_{\alpha_{\rho}} \rightarrow Q \rightarrow 1
$$

Let $G$ act on a compact space $X$ in such a way that $A$ acts trivially on $X$. We can see $X$ as a $\widetilde{Q}_{\alpha_{\rho}}$ space on which the central factor $\mathbb{S}^{1}$ acts trivially. Suppose that $p: E \rightarrow X$ is a $(G, \rho)$-equivariant vector bundle; that is, $p: E \rightarrow X$ is a $G$ equivariant vector bundle such that $\mathbb{V}_{\rho} \otimes \operatorname{Hom}_{A}\left(\mathbb{V}_{\rho}, E\right) \cong E$ as $A$-vector bundles. In general the vector $\operatorname{Hom}_{A}\left(\mathbb{V}_{\rho}, E\right)$ does not have a structure of a $G$-equivariant vector bundle that is compatible the $G$-structure on $E$. Instead, we are going to show in the next theorem that $\operatorname{Hom}_{A}\left(\mathbb{V}_{\rho}, E\right)$ has the structure of a $\widetilde{Q}_{\alpha_{\rho}}$-vector bundle on which the central factor $\mathbb{S}^{1}$ acts by multiplication of scalars. This is a key step in our work.

Theorem 2.5. Let $X$ be an $G$-space such that $A$ acts trivially on $X$. Assume that $g \cdot \rho \cong \rho$ for every $g \in G$. If $p: E \rightarrow X$ is a $(G, \rho)$-equivariant vector bundle, then 
$\operatorname{Hom}_{A}\left(\mathbb{V}_{\rho}, E\right)$ has the structure of a $\widetilde{Q}_{\alpha_{\rho}}$-vector bundle on which the central factor $\mathbb{S}^{1}$ acts by multiplication of scalars. Moreover, the assignment

$$
[E] \mapsto\left[\operatorname{Hom}_{A}\left(\mathbb{V}_{\rho}, E\right)\right]
$$

defines a natural one to one correspondence between isomorphism classes of $(G, \rho)$ equivariant vector bundles over $X$ and isomorphism classes of $\widetilde{Q}_{\alpha_{\rho}}$-equivariant vector bundles over $X$ for which the central $\mathbb{S}^{1}$ acts by multiplication of scalars.

Proof. Suppose that $p: E \rightarrow X$ is a $G$-vector bundle. Then $\operatorname{Hom}_{A}\left(\mathbb{V}_{\rho}, E\right)$ is a nonequivariant vector bundle over $X$. Next we give $\operatorname{Hom}_{A}\left(\mathbb{V}_{\rho}, E\right)$ an action of $\widetilde{Q}_{\alpha_{\rho}}$. For this suppose that $f \in \operatorname{Hom}_{A}\left(\mathbb{V}_{\rho}, E\right)_{x}$. If $q \in Q$ we define $q \bullet f \in \operatorname{Hom}_{A}\left(\mathbb{V}_{\rho}, E\right)_{q \cdot x}$ by

$$
(q \bullet f)(v)=\sigma(q) \cdot f\left(M_{q}^{-1} v\right),
$$

where $M_{q} \in U\left(V_{\rho}\right)$ is the element chosen above. It is easy to see that with this definition $q \bullet f$ is $A$-equivariant and that the map

$$
\begin{gathered}
q \bullet: \operatorname{Hom}_{A}\left(\mathbb{V}_{\rho}, E\right) \rightarrow \operatorname{Hom}_{A}\left(\mathbb{V}_{\rho}, E\right) \\
f \mapsto q \bullet f .
\end{gathered}
$$

is continuous. Moreover, if $q_{1}, q_{2} \in Q$ we have

$$
q_{1} \bullet\left(q_{2} \bullet f\right)=\alpha_{\rho}\left(q_{1}, q_{2}\right)\left(\left(q_{1} q_{2}\right) \bullet f\right) .
$$

Equation (2.6) allows us to define an action of $\widetilde{Q}_{\alpha_{\rho}}$ on $\operatorname{Hom}_{A}\left(\mathbb{V}_{\rho}, E\right)$ as follows. If $(q, z) \in \widetilde{Q}_{\alpha_{\rho}}$ and $f \in \operatorname{Hom}_{A}\left(\mathbb{V}_{\rho}, E\right)_{x}$ define

$$
(q, z) \cdot f:=z(q \bullet f) .
$$

Thus if $v \in V_{\rho}$ then

$$
((q, z) \cdot f)(v)=z\left(\sigma(q) \cdot f\left(M_{q}^{-1} v\right)\right)=\sigma(q) \cdot\left(z f\left(M_{q}^{-1} v\right)\right) .
$$

It can be checked that this defines an action of $\widetilde{Q}_{\alpha_{\rho}}$ on $\operatorname{Hom}_{A}\left(\mathbb{V}_{\rho}, E\right)$. Also, this action is fiberwise linear and the map $p: \operatorname{Hom}_{A}\left(\mathbb{V}_{\rho}, E\right) \rightarrow X$ is $\widetilde{Q}_{\alpha_{\rho}}$-equivariant so that $\operatorname{Hom}_{A}\left(\mathbb{V}_{\rho}, E\right)$ is a $\widetilde{Q}_{\alpha_{\rho}}$-equivariant vector bundle over $X$. By definition the central factor $\mathbb{S}^{1}$ acts by multiplication of scalars.

Suppose now that $p: F \rightarrow X$ is a $\widetilde{Q}_{\alpha_{\rho}}$-equivariant vector bundle over $X$ for which the central $\mathbb{S}^{1}$ acts by multiplication of scalars. Given $g \in G, f \in F_{x}$ and $v \in V_{\rho}$ define

$$
g \cdot(v \otimes f):=\left(M_{\pi(g)} \rho\left(\sigma(\pi(g))^{-1} g\right) v\right) \otimes((\pi(g), 1) \cdot f) \in\left(\mathbb{V}_{\rho} \otimes F\right)_{\pi(g) \cdot x}
$$

Using the cocycle identities it can be verified that this defines an action of $G$ on $\mathbb{V}_{\rho} \otimes F$, and since it is linear on the fibers, the bundle $\mathbb{V}_{\rho} \otimes F$ becomes a $G$-vector bundle. Moreover for $a \in A$, as $M_{1}=1$, we have that $a \cdot(v \otimes f)=(\rho(a) v) \otimes f$ so that $A$ acts on $\mathbb{V}_{\rho} \otimes F$ by the representation $\rho$; that is, $p: \mathbb{V}_{\rho} \otimes F \rightarrow X$ is a $(G, \rho)$-equivariant vector bundle over $X$.

Finally we need to show that this defines a one to one correspondence between isomorphism classes of $(G, \rho)$-equivariant vector bundles over $X$ and isomorphism classes of $\widetilde{Q}_{\alpha_{\rho}}$-equivariant vector bundles over $X$ for which the central $\mathbb{S}^{1}$ acts by 
multiplication of scalars. To this end, assume that $p: E \rightarrow X$ is a $(G, \rho)$-equivariant vector bundle over $X$, then by definition the map

$$
\begin{aligned}
\beta: \mathbb{V}_{\rho} \otimes \operatorname{Hom}_{A}\left(\mathbb{V}_{\rho}, E\right) & \rightarrow E \\
(v, f) & \mapsto f(v)
\end{aligned}
$$

is an isomorphism of $A$-vector bundles. Since $\operatorname{Hom}_{A}\left(\mathbb{V}_{\rho}, E\right)$ is a $\widetilde{Q}_{\alpha_{\rho}}$-equivariant vector bundle over $X$ on which the central $\mathbb{S}^{1}$ acts by multiplication of scalars, we may endow $\mathbb{V}_{\rho} \otimes \operatorname{Hom}_{A}\left(\mathbb{V}_{\rho}, E\right)$ with the structure of a $G$ vector bundle as it was done in equation (2.7). The map $\beta$ is an isomorphism of vector bundles and its $G$-equivariance follows from the next equations. For $g \in G$ we have

$$
\begin{aligned}
\beta(g \cdot(v \otimes f)) & =\beta\left(\left(M_{\pi(g)} \rho\left(\sigma(\pi(g))^{-1} g\right) v\right) \otimes((\pi(g), 1) \cdot f)\right) \\
& =\pi(g) \bullet f\left(M_{\pi(g)} \rho\left(\sigma(\pi(g))^{-1} g\right) v\right) \\
& =\sigma(\pi(g)) f\left(\rho\left(\sigma(\pi(g))^{-1} g\right) v\right)=g f(v) \\
& =g \beta(v \otimes f) .
\end{aligned}
$$

The previous argument shows that $\beta: \mathbb{V}_{\rho} \otimes \operatorname{Hom}_{A}\left(\mathbb{V}_{\rho}, E\right) \rightarrow E$ is an isomorphism of $G$-vector bundles.

Now, if $p: F \rightarrow X$ is a $\widetilde{Q}_{\alpha_{\rho}}$-equivariant vector bundle over $X$ for which the central $\mathbb{S}^{1}$ acts by multiplication of scalars, then by equation (2.7) we know that $\mathbb{V}_{\rho} \otimes F$ is a $(G, \rho)$-equivariant vector bundle. Let us show that $F$ and $\operatorname{Hom}_{A}\left(\mathbb{V}_{\rho}, \mathbb{V}_{\rho} \otimes\right.$ $F$ ) are isomorphic as $\widetilde{Q}_{\alpha_{\rho}}$-equivariant vector bundles. For this it can be showed in a similar way as it was done above that the canonical isomorphism of vector bundles

$$
\begin{aligned}
F & \rightarrow \operatorname{Hom}_{A}\left(\mathbb{V}_{\rho}, \mathbb{V}_{\rho} \otimes F\right) \\
x & \mapsto f_{x}: v \mapsto v \otimes x
\end{aligned}
$$

is $\widetilde{Q}_{\alpha_{\rho}}$-equivariant. Therefore the vector bundles $F$ and $\operatorname{Hom}_{A}\left(\mathbb{V}_{\rho}, \mathbb{V}_{\rho} \otimes F\right)$ are isomorphic as $\widetilde{Q}_{\alpha_{\rho}}$-equivariant vector bundles.

We conclude that the inverse map of the assignment $[E] \mapsto\left[\operatorname{Hom}_{A}\left(\mathbb{V}_{\rho}, E\right)\right.$ is precisely the map defined by the assignment $[F] \mapsto\left[\mathbb{V}_{\rho} \otimes F\right]$.

Theorem 2.5 provides a useful identification of the $(G, \rho)$-equivariant K-groups of Definition 2.2 with the $\alpha_{\rho}$-twisted $Q$-equivariant K-theory groups. For this purpose let us recall the definition of the $\alpha$-twisted $Q$-equivariant K-theory groups whenever $\alpha: Q \times Q \rightarrow \mathbb{S}^{1}$ is a 2-cocycle given in [2, Section 7].

Consider the $\mathbb{S}^{1}$-central extension of $Q$ that $\alpha$ defines

$$
1 \rightarrow \mathbb{S}^{1} \rightarrow \widetilde{Q}_{\alpha} \rightarrow Q \rightarrow 1
$$

with $\widetilde{Q}_{\alpha}$ as it is defined in (2.4). Let $X$ be a $Q$-space and endow it with the action of $\widetilde{Q}_{\alpha}$ induced by the $Q$ action. Let ${ }^{\alpha} K_{Q}^{0}(X)$ be Grothendieck group of the set of isomorphism classes of $\widetilde{Q}_{\alpha}$ vector bundles over $X$ on which $\mathbb{S}^{1}$ acts by multiplication of scalars on the fibers. For $n>0$ the twisted groups ${ }^{\alpha} K_{Q}^{n}(X)$ are defined as ${ }^{\alpha} \widetilde{K}_{Q}^{0}\left(\Sigma^{n} X_{+}\right)$. The groups ${ }^{\alpha} K_{Q}^{*}(X)$ are called the $\alpha$-twisted $Q$-equivariant K-theory groups of $X$. Note that ${ }^{\alpha} K_{Q}^{0}(X)$ is a free submodule of $K_{\widetilde{Q}_{\alpha}}^{*}(X)$ and we could have alternatively defined the $\alpha$-twisted $Q$-equivariant as this submodule. Furthermore 
note that the $\alpha$-twisted $Q$-equivariant vector bundles are the same as the $\left(\widetilde{Q}_{\alpha}, u\right)$ equivariant bundles where $u: \mathbb{S}^{1} \rightarrow U(1)$ is the irreducible representation given by the oriented isomorphisms of groups $u$ defined by multiplication by scalars.

Applying the definition of the $\alpha$-twisted $Q$-equivariant K-theory groups, Theorem 2.5] implies the following result which is the main result of this section:

Corollary 2.9. Let $X$ be a compact and Hausdorff $G$-space such that $A$ acts trivially on $X$. Assume furthermore that $\rho: A \rightarrow U\left(V_{\rho}\right)$ is a representation whose isomorphism class is fixed by $G$, i.e. $g \cdot \rho \cong \rho$ for every $g \in G$. Then the assignment

$$
\begin{aligned}
K_{G, \rho}^{*}(X) & \stackrel{\cong}{\rightrightarrows} \alpha_{\rho} K_{Q}^{*}(X) \\
{[E] } & \mapsto\left[\operatorname{Hom}_{A}\left(\mathbb{V}_{\rho}, E\right)\right]
\end{aligned}
$$

between the $(G, \rho)$-equivariant $K$-theory of $X$ and the $\alpha_{\rho}$-twisted $Q$-equivariant $K$ theory of $X$ is a natural isomorphism of $R(Q)$-modules.

\section{Decomposition formula in Equivariant K-Theory}

In this section we provide a decomposition of $K_{G}^{*}(X)$ whenever $G$ has a normal subgroup acting trivially on $X$. This decomposition is the main goal of this article.

Suppose that $G$ is a finite group for which we have a normal subgroup $A$. Let $Q=G / A$ so that we have a group extension

$$
1 \rightarrow A \stackrel{\iota}{\rightarrow} G \stackrel{\pi}{\rightarrow} Q \rightarrow 1 .
$$

Recall that the group $G$ acts on the set $\operatorname{Irr}(A)$ by conjugation. Notice that the group $A$ acts trivially on $\operatorname{Irr}(A)$ because if $x \in A$ and $\rho: A \rightarrow U\left(V_{\rho}\right)$ is a representation then $(x \cdot \rho)(a)=\rho(x)^{-1} \rho(a) \rho(x)$ for all $a \in A$ and thus $[x \cdot \rho]=[\rho]$ for $x \in A$. Therefore the action of $G$ on $\operatorname{Irr}(A)$ descends to an action of $Q$ on $\operatorname{Irr}(A)$ and the orbits of the action of $G$ on $\operatorname{Irr}(A)$ correspond to the orbits of the action of $Q$ on $\operatorname{Irr}(A)$. If $\rho: A \rightarrow U\left(V_{\rho}\right)$ is an irreducible representation we can consider the group $Q_{[\rho]}$ which is the isotropy subgroup of the action of $Q$ at $[\rho]$. Let $G_{[\rho]}=\pi^{-1}\left(Q_{[\rho]}\right) \subset$ $G$. Notice that $G_{[\rho]}$ is precisely the subgroup of $G$ such that $g \cdot[\rho]=[\rho]$ and we have a group extension

$$
1 \rightarrow A \stackrel{\iota}{\rightarrow} G_{[\rho]} \stackrel{\pi}{\rightarrow} Q_{[\rho]} \rightarrow 1 .
$$

Assume that $G$ acts on a compact and Hausdorff space $X$ in such a way that $A$ acts trivially on $X$. Let $p: E \rightarrow X$ be a $G$-equivariant vector bundle. Since $A$ acts trivially on $X$ then each fiber of $E$ can be seen as an $A$-representation. We can give $E$ a Hermitian metric that is invariant under the action of $A$. As before we have a natural isomorphism of $A$-vector bundles

$$
\beta: \bigoplus_{[\tau] \in \operatorname{Irr}(A)} \mathbb{V}_{\tau} \otimes \operatorname{Hom}_{A}\left(\mathbb{V}_{\tau}, E\right) \rightarrow E .
$$

As pointed out before, in general each of the pieces $\mathbb{V}_{\tau} \otimes \operatorname{Hom}_{A}\left(\mathbb{V}_{\tau}, E\right)$ does not have the structure of a $G$-equivariant vector bundle. However, the previous decomposition can be used to obtain a decomposition of $E$ as a direct sum of $G$-vector bundles by considering the different orbits of the action of $Q$ on $\operatorname{Irr}(A)$. We claim the following theorem: 
Theorem 3.2. Suppose that $A \subset G$ is a normal subgroup and $X$ is a compact, Hausdorff $G$-space on which $A$ acts trivially. Then there is a natural isomorphism

$$
\begin{aligned}
\Psi_{X}: K_{G}^{*}(X) & \rightarrow \bigoplus_{[\tau] \in G \backslash \operatorname{Irr}(A)} K_{G_{[\tau]}, \tau}^{*}(X) \\
E & \mapsto \bigoplus_{[\tau] \in G \backslash \operatorname{Irr}(A)} \mathbb{V}_{\tau} \otimes \operatorname{Hom}_{A}\left(\mathbb{V}_{\tau}, E\right)
\end{aligned}
$$

where $[\tau]$ runs over the orbits of $G$ on $\operatorname{Irr}(A)$ and $G_{[\tau]}$ is the isotropy group of $[\tau]$. This isomorphism is functorial on maps $X \rightarrow Y$ of $G$-spaces on which $A$ acts trivially.

Proof. Decompose the set $\operatorname{Irr}(A)$ in the form $\operatorname{Irr}(A)=\mathcal{A}_{1} \sqcup \mathcal{A}_{1} \sqcup \cdots \sqcup \mathcal{A}_{k}$, where $\mathcal{A}_{1}, \mathcal{A}_{2}, \ldots, \mathcal{A}_{k}$ are the different $G$-orbits on $\operatorname{Irr}(A)$, i.e. $G \backslash \operatorname{Irr}(A)=\left\{\mathcal{A}_{1}, \mathcal{A}_{2}, \ldots, \mathcal{A}_{k}\right\}$. For every $1 \leq i \leq k$ define

$$
E_{\mathcal{A}_{i}}=\bigoplus_{[\tau] \in \mathcal{A}_{i}} \mathbb{V}_{\tau} \otimes \operatorname{Hom}_{A}\left(\mathbb{V}_{\tau}, E\right)
$$

Notice that each $E_{\mathcal{A}_{i}}$ is an $A$-equivariant vector bundle over $X$, and moreover the map

$$
\beta: \bigoplus_{i=1}^{k} E_{\mathcal{A}_{i}}=\bigoplus_{[\tau] \in \operatorname{Irr}(A)} \mathbb{V}_{\tau} \otimes \operatorname{Hom}_{A}\left(\mathbb{V}_{\tau}, E\right) \rightarrow E
$$

defines an isomorphism of $A$-vector bundles. We will show that each $E_{\mathcal{A}_{i}}$ is a $G$-vector bundle and that the map $\beta$ is $G$-equivariant.

Let us fix $1 \leq i \leq k$ and an irreducible representation $\rho: A \rightarrow U\left(V_{\rho}\right)$ such that $[\rho] \in \mathcal{A}_{i}$. Therefore all the representations in $\mathcal{A}_{i}$ are of the form $[g \cdot \rho]$. Fix representatives $g_{1}=1, g_{2}, \ldots, g_{n_{i}}$ of the different cosets in $G / G_{[\rho]}$; that is, $G=\bigsqcup_{j=1}^{n_{i}} g_{j} G_{[\rho]}$. Therefore

$$
E_{\mathcal{A}_{i}}=\bigoplus_{j=1}^{n_{i}} \mathbb{V}_{g_{j} \cdot \rho} \otimes \operatorname{Hom}_{A}\left(\mathbb{V}_{g_{j} \cdot \rho}, E\right) .
$$

We first notice that $\mathbb{V}_{\rho} \otimes \operatorname{Hom}_{A}\left(\mathbb{V}_{\rho}, E\right)$ has the structure of a $G_{[\rho]}$-vector bundle. For this suppose that $h \in G_{[\rho]}$ and $v \otimes f \in\left(\mathbb{V}_{\rho} \otimes \operatorname{Hom}_{A}\left(\mathbb{V}_{\rho}, E\right)\right)_{x}$. A first candidate for the action of $h$ on $v \otimes f$ is the element $v \otimes\left(c_{h} \circ f\right) \in \mathbb{V}_{h \cdot \rho} \otimes \operatorname{Hom}_{A}\left(\mathbb{V}_{h \cdot \rho}, E\right)$, where $c_{h} \circ f(w)=h f(w)$. This does not define an action of $G_{[\rho]}$ as we land in a different (but isomorphic) vector bundle. We can correct this in the following way. By definition for every $h \in G_{[\rho]}$ we have $h \cdot \rho \cong \rho$. Therefore for every $h \in G_{[\rho]}$ we can choose some element $M_{h} \in U\left(V_{\rho}\right)$ such that $\rho\left(h^{-1} a h\right)=M_{h}^{-1} \rho(a) M_{h}$ for all $a \in A$ and we choose $M_{a}=\rho(a)$ for all $a \in A$. For $h \in G_{[\rho]}$ and $v \otimes f \in \mathbb{V}_{\rho} \otimes \operatorname{Hom}_{A}\left(\mathbb{V}_{\rho}, E\right)$ we define

$$
h \star(v \otimes f)=\left(M_{h} v\right) \otimes(h \bullet f),
$$

where $(h \bullet f)(w)=h f\left(M_{h}^{-1} w\right)$. In a similar way as in equations (2.8) it can be checked that this defines a structure of a $G_{[\rho]}$-vector bundle on $\mathbb{V}_{\rho} \otimes \operatorname{Hom}_{A}\left(\mathbb{V}_{\rho}, E\right)$ in such a way that the evaluation map

$$
\beta: \mathbb{V}_{\rho} \otimes \operatorname{Hom}_{A}\left(\mathbb{V}_{\rho}, E\right) \rightarrow E, \quad v \otimes f \mapsto f(v)
$$

is a $G_{[\rho]}$-equivariant isomorphism onto its image (we may take $M_{h}$ as the transformation $M_{\pi(h)} M_{\sigma(\pi(h))^{-1} h}$ defined in equations (2.8)). With this in mind we can 
define an action of $G$ on $E_{\mathcal{A}_{i}}$ in the following way. Suppose that $g \in G$ and that $v \otimes f \in\left(\mathbb{V}_{g_{j} \cdot \rho} \otimes \operatorname{Hom}_{A}\left(\mathbb{V}_{g_{j} \cdot \rho}, E\right)\right)_{x}$. Decompose $g g_{j}$ in the form $g g_{j}=g_{l} h$, where $1 \leq l \leq n_{i}$ and $h \in G_{[\rho]}$. In other words $g_{l}$ is the representative chosen for the coset $\left(g g_{j}\right) G_{[\rho]}$ and $h=g_{l}^{-1} g g_{j}$. Define

$$
g \star(v \otimes f):=\left(M_{h} v\right) \otimes(g \bullet f) \in\left(\mathbb{V}_{g_{l} \cdot \rho} \otimes \operatorname{Hom}_{A}\left(\mathbb{V}_{g_{l} \cdot \rho}, E\right)\right)_{g x},
$$

where $(g \bullet f)(w)=g f\left(M_{h}^{-1} w\right)$. Let us show that this defines an action of $G \in E_{\mathcal{A}_{i}}$.

We show first that $\left.g \bullet f \in \operatorname{Hom}_{A}\left(\mathbb{V}_{g_{l} \cdot \rho}, E\right)\right)_{g x}$ when $\left.f \in \operatorname{Hom}_{A}\left(\mathbb{V}_{g_{j} \cdot \rho}, E\right)\right)_{x}$. To see this recall that the representation $\mathbb{V}_{g_{j} \cdot \rho}$ has as underlying spce $V_{\rho}$ and the action of $A$ is given by $a \cdot w=\rho\left(g_{j}^{-1} a g_{j}\right) w$. Therefore, the fact that $\left.f \in \operatorname{Hom}_{A}\left(\mathbb{V}_{g_{j} \cdot \rho}, E\right)\right)_{x}$ means that for all $a \in A$ and all $w \in V_{g_{j} \cdot \rho}$ we have

$$
f\left(\rho\left(g_{j}^{-1} a g_{j}\right) w\right)=a f(w) .
$$

With this in mind, for all $w \in V_{g_{l} \cdot \rho}$ and all $a \in A$ we have

$$
\begin{aligned}
(g \bullet f)(a \cdot w) & =(g \bullet f)\left(\rho\left(g_{l}^{-1} a g_{l}\right) w\right)=g f\left(M_{h}^{-1} \rho\left(g_{l}^{-1} a g_{l}\right) w\right) \\
& =g f\left(\left(M_{h}^{-1} \rho\left(g_{l}^{-1} a g_{l}\right) M_{h}\right) M_{h}^{-1} w\right)=g f\left(\rho\left(h^{-1} g_{l}^{-1} a g_{l} h\right) M_{h}^{-1} w\right) \\
& =g f\left(\rho\left(g_{j}^{-1} g^{-1} a g g_{j}\right) M_{h}^{-1} w\right)=g\left(g^{-1} a g\right) f\left(M_{h}^{-1} w\right) \\
& =a(g \bullet f)(w) .
\end{aligned}
$$

Therefore $g \bullet f \in \operatorname{Hom}_{A}\left(\mathbb{V}_{g_{l} \cdot \rho}, E\right)_{g x}$. On the other hand, notice that if $g \in G$ and $v \otimes f \in\left(\mathbb{V}_{g_{j} \cdot \rho} \otimes \operatorname{Hom}_{A}\left(\mathbb{V}_{g_{j} \cdot \rho}, E\right)\right)_{x}$ then

$$
\beta(g \star(v \otimes f))=g f\left(M_{h}^{-1} M_{h} v\right)=g f(v)=g \beta(f \otimes v) .
$$

Since the evaluation map $\beta$ is injective and preserves the $G$-action, then it follows that for every $g_{1}, g_{2} \in G$ we have $g_{1} \star\left(g_{2} \star(v \otimes f)\right)=\left(g_{1} g_{2}\right) \star(v \otimes f)$. The above argument proves that for each $1 \leq i \leq k$ the vector bundle $E_{\mathcal{A}_{i}}$ has the structure of $G$-vector bundle. Moreover, the map

$$
\beta: \bigoplus_{i=1}^{k} E_{\mathcal{A}_{i}} \rightarrow E
$$

is an isomorphism of $A$-vector and the map $\beta$ is $G$-equivariant so that $\beta$ is an isomorphism of $G$-vector bundles.

Now, if for each $\mathcal{A}_{i}$ we choose a representation $\left[\tau_{i}\right] \in \mathcal{A}_{i}$ we may write the map $\Psi_{X}$ as the direct sum $\bigoplus_{i=1}^{k} \Psi_{X}^{i}$ of the maps

$$
\Psi_{X}^{i}: K_{G}^{*}(X) \rightarrow K_{G_{\left[\tau_{i}\right]}, \tau_{i}}(X), \quad E \mapsto \mathbb{V}_{\tau_{i}} \otimes \operatorname{Hom}_{A}\left(\mathbb{V}_{\tau_{i}}, E\right) .
$$

Note that $\Psi_{X}^{i}\left(E_{\mathcal{A}_{j}}\right)=0$ for $i \neq j$.

Let us construct the map $K_{G_{\left[\tau_{i}\right]}, \tau_{i}}(X) \rightarrow K_{G}^{*}(X)$ which will be the right inverse of $\Psi_{X}^{i}$.

Let $\rho=\tau_{i}$ and consider a vector bundle $F \in \operatorname{Vec}_{G_{[\rho]}, \rho}(X)$. We need to construct a $G$-vector bundle from $F$ taking into account that $G$ acts on $X$.

For $g \in G$ let $g^{*} F:=\{(x, f) \in X \times F \mid g x=\pi f\}$ be the pullback bundle where $\pi: F \rightarrow X$ is the projection map. Consider the bundle

$$
\bigoplus_{j=1}^{n}\left(g_{j}^{-1}\right)^{*} F
$$


where $g_{1}=1, g_{2}, \ldots, g_{n}$ are fixed elements of the different cosets in $G / G_{[\rho]}$. Endow $\bigoplus_{j=1}^{n}\left(g_{j}^{-1}\right)^{*} F$ with a $G$ action in the following way. For $(x, f) \in\left(g_{j}^{-1}\right)^{*} F$ and $g \in G$, let $g_{l} \in G$ and $h \in G_{[\rho]}$ be such that $g g_{j}=g_{l} h$. Define the action of $g$ as follows:

$$
g \circ(x, f):=(g x, h f) \in\left(g_{l}^{-1}\right)^{*} F .
$$

For another $\bar{g} \in G$, let $g_{m} \in G$ and $e \in G_{[\rho]}$ such that $\bar{g} g g_{j}=g_{m} e$ and hence $\bar{g} g_{l}=g_{m} e h^{-1}$. Then we have the following equalities:

$$
\bar{g} \circ(g \circ(x, f))=\bar{g} \circ(g x, h f)=\left(\bar{g} g x, e h^{-1} h f\right)=(\bar{g} g x, e f)=\bar{g} g \circ(x, f)
$$

which imply that $\bigoplus_{j=1}^{n}\left(g_{j}^{-1}\right)^{*} F$ is a $G$-vector bundle compatible with the $G$ action on $X$.

Note that for $h \in G_{[\rho]}$ and $(x, f) \in\left(g_{j}^{-1}\right)^{*} F$ we have that

$$
g_{j} h g_{j}^{-1} \circ(x, f)=\left(g_{j} h g_{j}^{-1} x, h f\right),
$$

and therefore for $k=g_{j} h g_{j}^{-1} \in G_{g_{j} \cdot[\rho]}$ we have $k \circ(x, f)=\left(k x, g_{j}^{-1} k g_{j} f\right)$. This implies that the restricted action of $G_{g_{j} \cdot[\rho]} \subset G$ on $\left(g_{j}^{-1}\right)^{*} F$ matches the conjugation action that can be defined on the the bundle $\left(g_{j}^{-1}\right)^{*} F$; in particular the restriction of the $G_{[\rho]^{-a c t i o n}}$ on $1^{*} F$ matches the original action on $F$.

Now, since we have that $\mathbb{V}_{\rho} \otimes \operatorname{Hom}_{A}\left(\mathbb{V}_{\rho}, \bigoplus_{j=1}^{n}\left(g_{j}^{-1}\right)^{*} F\right) \cong F$ as $G_{[\rho]^{-}}$-vector bundles, we have that at the level of K-theory we obtain that

$$
\Psi_{X}\left(\bigoplus_{j=1}^{n}\left(g_{j}^{-1}\right)^{*} F\right)=F \in \operatorname{Vec}_{G_{[\rho]}, \rho}(X) .
$$

Therefore we have that the maps $\Psi_{X}^{i}$ have right inverses, and hence we conclude that the map $\Psi_{X}$ is indeed an isomorphism.

The functoriality follows from the fact that the bundles $\mathbb{V}_{\tau} \otimes \operatorname{Hom}_{A}\left(\mathbb{V}_{\tau}, f^{*} E\right)$ and $f^{*}\left(\mathbb{V}_{\tau} \otimes \operatorname{Hom}_{A}\left(\mathbb{V}_{\tau}, E\right)\right)$ are canonically isomorphic as $\left(G_{[\tau]}, \tau\right)$-equivariant bundles whenever $f: Y \rightarrow X$ is a $G$-equivariant map from spaces on which $A$ acts trivially.

Theorem 3.2 and Corollary 2.9 imply the main result of this article.

Theorem 3.4. Suppose that $A \subset G$ is a normal subgroup and $X$ is a compact, Hausdorff $G$-space on which $A$ acts trivially. Then there is a natural isomorphism

$$
\begin{aligned}
\Psi_{X}: K_{G}^{*}(X) & \rightarrow \bigoplus_{[\tau] \in G \backslash \operatorname{Irr}(A)}{ }^{\alpha_{\tau}} K_{Q_{[\tau]}}^{*}(X) \\
E & \mapsto \bigoplus_{[\tau] \in G \backslash \operatorname{Irr}(A)} \operatorname{Hom}_{A}\left(\mathbb{V}_{\tau}, E\right) .
\end{aligned}
$$

This isomorphism is functorial on maps $X \rightarrow Y$ of $G$-spaces on which $A$ acts trivially.

Proof. The result follows from the fact that the canonical map

$$
\operatorname{Hom}_{A}\left(\mathbb{V}_{\tau}, E\right) \cong \operatorname{Hom}_{A}\left(\mathbb{V}_{\tau}, \mathbb{V}_{\tau} \otimes \operatorname{Hom}_{A}\left(\mathbb{V}_{\tau}, E\right)\right)
$$

induces an isomorphism of $\alpha_{\tau}$-twisted $Q_{[\tau]}$-equivariant bundles. 
As a particular application of the previous theorem, suppose that $X$ is a compact space on which $Q$ acts freely. Then we can see $X$ as a $G$-space in such a way that for every $x \in X$ we have that $G_{x}=A$. In this particular case the twisted equivariant groups ${ }^{\alpha_{\tau}} K_{Q_{[\tau]}}^{*}(X)$ that appear in the previous theorem can be seen as suitable non-equivariant twisted K-groups as is explained next. For this suppose that $\mathcal{H}$ is a separable infinite dimensional Hilbert space. Let $P U(\mathcal{H})$ denote the projective unitary group with the strong operator topology. Given a space $Y$ together with a continuous function $f: Y \rightarrow B P U(\mathcal{H})$, pulling back the universal principal $P U(\mathcal{H})$-bundle $\operatorname{EPU}(\mathcal{H}) \rightarrow B P U(\mathcal{H})$ along $f$, we obtain a principal $P U(\mathcal{H})$-bundle $P_{f} \rightarrow Y$. Associated to the pair $(Y ; f)$ we may define the twisted K-theory groups

$$
K^{-p}(Y ; f):=\pi_{p}\left(\Gamma\left(\operatorname{Fred}\left(P_{f}\right)\right)\right)
$$

as the homotopy groups of the space of sections of the associated Fredholm bundle $\operatorname{Fred}\left(P_{f}\right):=P_{f} \times_{P U(\mathcal{H})} \operatorname{Fred}(\mathcal{H})$. This is the usual definition of non-equivariant twisted K-groups given in [5, Def. 3.3].

Suppose now that $\alpha: Q \times Q \rightarrow \mathbb{S}^{1}$ is a 2-cocycle and let

$$
1 \rightarrow \mathbb{S}^{1} \rightarrow \widetilde{Q}_{\alpha} \rightarrow Q \rightarrow 1
$$

be the central extension that $\alpha$ defines as in (2.4). Let $\mathcal{H}_{\widetilde{Q}_{\alpha}}$ be a separable Hilbert space endowed with a continuous linear action of $\widetilde{Q}_{\alpha}$ such that all representations of $\widetilde{Q}_{\alpha}$ appear infinitely many times. The Hilbert space $\mathcal{H}_{\widetilde{Q}_{\alpha}}$ splits into isotypical components relative to the action of $\mathbb{S}^{1}$

$$
\mathcal{H}_{\widetilde{Q}_{\alpha}} \cong \bigoplus_{k \in \mathbb{Z}} \mathcal{H}_{\widetilde{Q}_{\alpha}}^{k}
$$

with $\mathcal{H}_{\widetilde{Q}_{\alpha}}^{k}$ being the eigenspace of the degree $k$ map. The eigenspace $\mathcal{H}:=\mathcal{H}_{\widetilde{Q}_{\alpha}}^{1}$ is a $\widetilde{Q}_{\alpha}$ representation on which $\mathbb{S}^{1}$ acts by multiplication of scalars and all the representations of this kind appear infinitely number of times. Hence we have an induced homomorphism of groups $\widetilde{\phi}_{\alpha}: \widetilde{Q}_{\alpha} \rightarrow U(\mathcal{H})$ which induces a homomorphism $\phi_{\alpha}: Q \rightarrow P U(\mathcal{H})$ making the following diagram of group extensions commutative

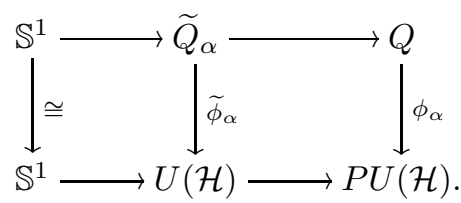

Using the homomorphism $\phi_{\alpha}: Q \rightarrow P U(\mathcal{H})$ and the natural action of $P U(\mathcal{H})$ on $\operatorname{Fred}(\mathcal{H})$ we can obtain an action of $Q$ on $\operatorname{Fred}(\mathcal{H})$. Recall that the $\alpha$-twisted $Q$ equivariant K-theory groups are generated by the $\widetilde{Q}_{\alpha}$-equivariant vector bundles over $X$ on which $\mathbb{S}^{1}$ acts by multiplication of scalars, therefore using the alternative definition of equivariant $K$-theory using Fredholm operators, the group ${ }^{\alpha} K_{Q}^{*}(X)$ may be alternatively defined as the homotopy groups of the space

$$
\operatorname{map}(X, \operatorname{Fred}(\mathcal{H}))^{Q}=\left\{f: X \rightarrow \operatorname{Fred}(\mathcal{H}) \mid f(q \cdot x)=\phi_{\alpha}(q) \cdot f(x)\right\}
$$

of $Q$-equivariant maps from $X$ to $\operatorname{Fred}(\mathcal{H})$, i.e.

$$
{ }^{\alpha} K_{Q}^{-p}(X) \cong \pi_{p}\left(\operatorname{map}(X, \operatorname{Fred}(\mathcal{H}))^{Q}\right) .
$$


In the particular case on which $Q$ acts freely on $X$ there is an homeomorphism of topological spaces

(3.6) $\operatorname{map}(X, \operatorname{Fred}(\mathcal{H}))^{Q} \stackrel{\cong}{\rightrightarrows} \Gamma\left(X \times_{Q} \operatorname{Fred}(\mathcal{H}) \rightarrow X / Q\right) \quad f \mapsto([f]:[x] \mapsto[x, f(x)])$

between the space of $Q$-equivariant maps from $X$ to $\operatorname{Fred}(\mathcal{H})$ and the space of sections of the $\operatorname{Fred}(\mathcal{H})$-bundle $X \times{ }_{Q} \operatorname{Fred}(\mathcal{H}) \rightarrow X / Q$.

On the other hand, since $X$ is a free $Q$-space there is a unique up to homotopy $Q$-equivariant map $X \rightarrow E Q$ inducing a map $h: X / G \rightarrow B Q$ at the level of the quotient spaces. Combining $h$ with the map $B \phi_{\alpha}$ we obtain the following commutative diagram

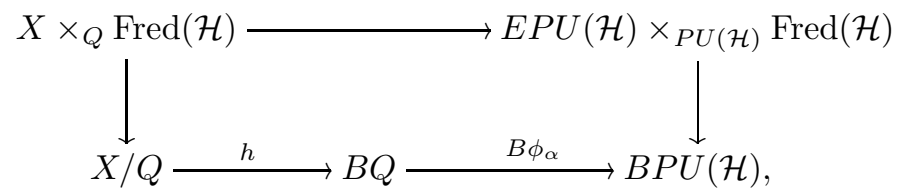

where the outer square is a pullback square. Therefore

$$
K^{-p}\left(X / Q, B \phi_{\alpha} \circ h\right) \cong \pi_{p}\left(\Gamma\left(X \times{ }_{Q} \operatorname{Fred}(\mathcal{H}) \rightarrow X / Q\right)\right) .
$$

Using (3.5), (3.6) and (3.7) we conclude that if $Q$ acts freely on $X$, the $\alpha$-twisted $Q$-equivariant K-theory of $X$ is canonically isomorphic to the twisted K-theory of the pair $\left(X / Q, B \phi_{\alpha} \circ h\right)$, i.e.

$$
{ }^{\alpha} K_{Q}^{*}(X) \cong K^{*}\left(X / Q, B \phi_{\alpha} \circ h\right) .
$$

Note that the cohomology class $\left[\alpha^{\mathbb{Z}}\right] \in H^{3}(B Q, \mathbb{Z})$ that $\alpha$ defines, is the pullback of the generator of $H^{3}(B P U(\mathcal{H}), \mathbb{Z})=\mathbb{Z}$ under the classifying map $B \phi_{\alpha}$. Hence $h^{*}\left[\alpha^{\mathbb{Z}}\right] \in H^{3}(X / Q, \mathbb{Z})$ is the pullback under $B \phi_{\alpha} \circ h$ of the generator of $H^{3}(B P U(\mathcal{H}), \mathbb{Z})$. The cohomology class $h^{*}\left[\alpha^{\mathbb{Z}}\right]$ classifies the isomorphism class of the projective unitary bundle over $X / Q$ which $\phi_{\alpha}$ induces.

Combining (3.8) with Theorem 3.2 we obtain:

Theorem 3.9. Let $A$ be a normal subgroup of a finite group $G$ and denote $Q=$ $G / A$. Let $X$ be a free $Q$-space and consider it as a $G$-space on which $A$ acts trivially. Then there is a natural decomposition of the $G$-equivariant $K$-theory of $X$ into a direct sum of twisted $K$-theories in the following way

$$
\Psi_{X}: K_{G}^{*}(X) \rightarrow \bigoplus_{[\rho] \in Q \backslash \operatorname{Irr}(A)} K^{*}\left(X / Q_{[\rho]} ; B \phi_{\alpha_{\rho}} \circ h_{\rho}\right),
$$

where $\phi_{\alpha_{\rho}}: Q_{[\rho]} \rightarrow P U(\mathcal{H})$ is the stable homomorphism defined by $\alpha_{\rho}$ and $h_{\rho}:$ $X / Q_{[\rho]} \rightarrow B Q_{[\rho]}$ is the classifying map of the $Q_{[\rho]}$-principal bundle $X \rightarrow X / Q_{[\rho]}$.

\section{The Decomposition formula and the Completion Theorem}

Let $A$ be a normal subgroup in $G$ and $Q=G / A$. Suppose that $X$ a $G$-space on which $A$ acts trivially. Let $E_{Q}^{n}=Q * Q * \cdots * Q$ be the Milnor join of $n$-copies of $Q$ thus making $E Q$ the direct limit of the free $Q$-spaces $E_{Q}^{n}$. Let $B_{Q}^{n}=E_{Q}^{n} / Q$ and note that it is the union of $n$-contractible open sets, thus the product of any $n$ elements in the reduced K-theory groups $\widetilde{K}^{*}\left(B_{Q}^{n}\right)$ is zero.

Consider the ideal $I(Q)=\operatorname{ker}\left(\operatorname{res}_{A}^{G}: R(G) \rightarrow R(A)\right)$ of virtual representations whose restriction to $A$ vanish, and consider the ideals $I_{Q}^{n}=\operatorname{ker}\left(R(G) \rightarrow K_{G}\left(E_{Q}^{n}\right)\right)$ of the map that takes a representation $V$ an maps it to $V \times E_{Q}^{n}$. Consider the map 
$K_{G}\left(E_{Q}^{n}\right) \rightarrow R(A)^{G}$ induced by restricting a $G$-equivariant vector bundle to a point, and note that the restriction of representations is the composition of the maps

$$
R(G) \rightarrow K_{G}\left(E_{Q}^{n}\right) \rightarrow R(A)^{G} .
$$

This implies that $I_{Q}^{n} \subset I(Q)$ and we may define the completion of $K_{G}^{*}(X)$ on the ideals $I_{Q}^{n}$ by the inverse limit:

$$
\left.\widehat{K_{G}^{*}(X)}\right)_{I_{Q}}=\lim _{\underline{n}} K_{G}^{*}(X) /\left(I_{Q}^{n} \cdot K_{G}^{*}(X)\right) .
$$

Let $K_{G}^{*}(X \times E Q)=\lim _{\leftarrow} K_{G}^{*}\left(X \times E_{Q}^{n}\right)$ and consider the canonical map

$$
K_{G}^{*}(X) \rightarrow K_{G}^{*}(X \times E Q)
$$

induced by the pullbacks $K_{G}^{*}(X) \rightarrow K_{G}^{*}\left(X \times E_{Q}^{n}\right)$ of the projection maps $X \times E_{Q}^{n} \rightarrow$ $X$. The generalization of the Completion Theorem of Atiyah and Segal [4, Thm. 2.1] to families of subgroups [8, Thm. 2.1] states that the induced map at the level of the completion

$$
\left.\widehat{K_{G}^{*}(X)}\right)_{I_{Q}} \cong K_{G}^{*}(X \times E Q)
$$

is an isomorphism. Using Theorem 3.2 we may define the completed groups

$$
\widehat{K_{G_{\left[\tau_{i}\right]}^{*}, \tau_{i}}^{*}}(X)_{I_{Q}}:=\lim _{\leftarrow} K_{G_{\left[\tau_{i}\right]}, \tau_{i}}^{*}(X) / \Psi_{X}^{i}\left(I_{Q}^{n} \cdot K_{G}(X)\right)
$$

where $\left[\tau_{i}\right] \in G \backslash \operatorname{Irr}(A)$ and

$$
\Psi_{X}^{i}: K_{G}^{*}(X) \rightarrow K_{G_{\left[\tau_{i}\right]}, \tau_{i}}(X), \quad E \mapsto \mathbb{V}_{\tau_{i}} \otimes \operatorname{Hom}_{A}\left(\mathbb{V}_{\tau_{i}}, E\right)
$$

is the projection map of Theorem 3.2 that appears in (3.3). Therefore we have that the completion theorem holds also component-wise.

Proposition 4.1. Let $X$ be a $G$ compact, Hausdorff space on which $A$ acts trivially. Let $Q=G / A$, take $\tau_{i} \in \operatorname{Irr}(A)$, consider the projection maps $X \times E_{Q}^{n} \rightarrow X$ and let

$$
K_{G_{\left[\tau_{i}\right]}, \tau_{i}}^{*}(X) \stackrel{\cong}{\rightrightarrows} K_{G_{\left[\tau_{i}\right]}, \tau_{i}}^{*}(X \times E Q)
$$

be the homomorphism that the projections define. Then the induced map

$$
\widehat{\widehat{K_{G_{\left[\tau_{i}\right]}, \tau_{i}}^{*}}}(X)_{I_{Q}} \stackrel{\cong}{\rightrightarrows} K_{G_{\left[\tau_{i}\right]}, \tau_{i}}^{*}(X \times E Q)
$$

is an isomorphism.

Proof. The result follows directly from Theorem 3.2 and the Completion Theorem [8, Thm. 2.1].

Applying Corollary 2.9 and the isomorphism obtained in (3.8) to the expression on the right, we obtain the canonical isomorphism

$$
\widehat{K_{G_{\left[\tau_{i}\right]}^{*}, \tau_{i}}}(X)_{I_{Q}} \stackrel{\cong}{\rightrightarrows} K^{*}\left(X \times_{Q_{\left[\tau_{i}\right]}} E Q ; B \phi_{\tau_{i}} \circ h_{\tau_{i}}\right)
$$

between the completed group of $K_{G_{\left[\tau_{i}\right]}, \tau_{i}}^{*}(X)$ and the $B \phi_{\tau_{i}} \circ h_{\tau_{i}}$-twisted K-theory of the space $X \times_{Q_{\left[\tau_{i}\right]}} E Q$. 


\section{Atiyah-Hirzebruch-Segal Spectral Sequence}

In this section we use Theorem 3.9 to describe the third differential of the generalized Atiyah-Hirzebruch spectral sequence developed by Segal in [9, §5] whenever we consider spaces with constant isotropy.

Assume that $G$ is a finite group acting on a compact $G$-CW complex $X$ in such a way that $G_{x}=A$ for every $x \in A$. Here $A$ is a normal subgroup of $G$ and $Q=G / A$. Let $R(-)$ denote the coefficient system defined by the assignment $G / H \mapsto R(H)$ with $R(H)$ the Grothendieck ring of complex representations of the group $H$. Since the action of $G$ on $X$ has constant isotropy then the Bredon cohomology groups $H_{G}^{p}(X, R(-))$ can be identified with the cohomology of the cochain complex $\operatorname{Hom}_{\mathbb{Z}[G]}\left(C_{*}(X), R(A)\right)$. The group $A$ acts trivially on both $C_{*}(X)$ and $R(A)$ so that this cochain complex is isomorphic to $\operatorname{Hom}_{\mathbb{Z}[Q]}\left(C_{*}(X), R(A)\right)$. As a $Q$-representation we have a decomposition $R(A) \cong \bigoplus_{[\rho] \in Q \backslash \operatorname{Irr}(A)} \mathbb{Z}\left[Q / Q_{[\rho]}\right]$. Using this decomposition we can identify $H_{G}^{p}(X, R(-))$ with $\bigoplus_{[\rho] \in Q \backslash \operatorname{Irr}(A)} H^{p}\left(X / Q_{[\rho]} ; \mathbb{Z}\right)$.

On the other hand, let $\mathcal{U}=\left\{U_{i}\right\}_{i \in \mathcal{I}}$ be an open cover of $X$ by $G$-invariant open sets. Assume that $\mathcal{I}$ is a well ordered set. We say that $\mathcal{U}$ s a contractible slice cover if for every sequence $i_{1} \leq \cdots \leq i_{p}$ of elements in $\mathcal{I}$ with $U_{i_{1}, \ldots, i_{p}}$ nonempty we can find some element $x_{i_{1}, \ldots, i_{p}} \in U_{i_{1}, \ldots, i_{p}}$ such that the inclusion map $G x_{i_{1}, \ldots, i_{p}} \hookrightarrow U_{i_{1}, \ldots, i_{p}}$ is a $G$-homotopy equivalence. Here we are using the notation $U_{i_{1}, \ldots, i_{p}}=U_{i_{1}} \cap \cdots \cap U_{i_{p}}$. It can be seen that such a contractible slice cover exists for any compact $G$-CW complex.

Let us consider the filtration of $K_{G}^{*}(X)$ that the open cover induces and the spectral sequence associated to this filtration; the definition and the properties of this spectral sequence were carried out by Segal in [9, §5]. The second page of the spectral sequence is the Bredon cohomology with coefficients in representations $H_{G}^{*}(X, R(-))$ and in our case this groups may be identified with

$$
E_{2}^{p, \text { even }} \cong \bigoplus_{[\rho] \in Q \backslash \operatorname{Irr}(A)} H^{p}\left(X / Q_{[\rho]} ; \mathbb{Z}\right) \text { and } E_{2}^{p, \text { odd }}=0 .
$$

By Theorem 3.9 we know that the $G$-equivariant K-theory of $X$ decomposes in a direct sum of twisted K-theories

$$
\Psi_{X}: K_{G}^{*}(X) \rightarrow \bigoplus_{[\rho] \in Q \backslash \operatorname{Irr}(A)} K^{*}\left(X / Q_{[\rho]} ; B \phi_{\alpha_{\rho}} \circ h_{\rho}\right),
$$

where $\left[\alpha_{\rho}\right] \in H^{2}\left(Q_{[\rho]}, \mathbb{S}^{1}\right)$ is the obstruction on lifting the representation $\rho$ to $G_{[\rho]}$, $\phi_{\alpha_{\rho}}: Q_{[\rho]} \rightarrow P U(\mathcal{H})$ is the stable homomorphism defined by $\alpha_{\rho}$ and $h_{\rho}: X / Q_{[\rho]} \rightarrow$ $B Q_{[\rho]}$ is the classifying map of the $Q_{[\rho]}$-principal bundle $X \rightarrow X / Q_{[\rho]}$.

The spectral sequence associated to $K_{G}^{*}(X)$ decomposes as a direct sum of the spectral sequences associated to the twisted K-theories $K^{*}\left(X / Q_{[\rho]} ; B \phi_{\alpha_{\rho}} \circ h_{\rho}\right)$. Therefore if we denote by $\left[\alpha_{\rho}^{\mathbb{Z}}\right] \in H^{3}\left(B Q_{[\rho]}, \mathbb{Z}\right)$ the element that $\left[\alpha_{\rho}\right]$ defines via the isomorphism

$$
H^{2}\left(B Q_{[\rho]}, \mathbb{S}^{1}\right) \cong H^{3}\left(B Q_{[\rho]}, \mathbb{Z}\right),
$$

then the cohomology class $h_{\rho}^{*}\left[\alpha_{\rho}^{\mathbb{Z}}\right] \in H^{3}\left(X / Q_{[\rho]}, \mathbb{Z}\right)$ is the one that the map $B \phi_{\alpha_{\rho}} \circ$ $h_{\rho}: X / Q_{[\rho]} \rightarrow B P U(\mathcal{H}) \simeq K(\mathbb{Z}, 3)$ defines. Therefore by [6. Proposition 4.6] the third differential in the Atiyah-Hirzebruch spectral sequence associated twisted 
$\mathrm{K}$-groups $K^{*}\left(X / Q_{[\rho]} ; B \phi_{\alpha_{\rho}} \circ h_{\rho}\right)$ is the operator

$$
\begin{aligned}
d_{3}^{\rho}: H^{*}\left(X / Q_{[\rho]} ; \mathbb{Z}\right) & \rightarrow H^{*+3}\left(X / Q_{[\rho]} ; \mathbb{Z}\right) \\
\eta & \mapsto d_{3}^{\rho}(\eta)=S q_{\mathbb{Z}}^{3} \eta-h_{\rho}^{*}\left[\alpha_{\rho}^{\mathbb{Z}}\right] \cup \eta .
\end{aligned}
$$

Here $S q_{\mathbb{Z}}^{3}$ is the composition of the maps $\beta \circ S q^{2} \circ \bmod _{2}$, where $\bmod _{2}$ is the reduction modulo $2, S q^{2}$ is the Steenrod operation, and $\beta$ is the Bockstein map. We obtain the following theorem.

Theorem 5.1. Suppose that $A \subset G$ is a normal subgroup and let $Q=G / A$. Let $X$ be a compact $G-C W$ complex such that $G_{x}=A$ for all $x \in X$. With the identifications made above, the third differential of the Atiyah-Hirzebruch spectral sequence

$$
d_{3}: \bigoplus_{[\rho] \in Q \backslash \operatorname{Irr}(A)} H^{p}\left(X / Q_{[\rho]} ; \mathbb{Z}\right) \rightarrow \bigoplus_{[\rho] \in Q \backslash \operatorname{Irr}(A)} H^{p}\left(X / Q_{[\rho]} ; \mathbb{Z}\right)
$$

is defined coordinate-wise in such a way that for $\eta \in H^{p}\left(X / Q_{[\rho]} ; \mathbb{Z}\right)$ we have

$$
d_{3}(\eta):=d_{3}^{\rho}(\eta)=S q_{\mathbb{Z}}^{3} \eta-h_{\rho}^{*}\left[\alpha_{\rho}^{\mathbb{Z}}\right] \cup \eta
$$

\section{EXAMPLES}

Let us take the dihedral group $G=D_{8}$ generated by the elements $a, b$ with relations $a^{4}=b^{2}=1$ and $b a b=a^{3}$. Let $\operatorname{Irr}\left(D_{8}\right)=\{\mathbf{1}, \lambda, \sigma, \sigma \otimes \lambda, \nu\}$ be the set of isomorphism classes of irreducible representations of $D_{8}$ defined by the following homomorphisms:

$$
\begin{aligned}
\lambda: D_{8} \rightarrow \mathbb{C}^{*}, \lambda(a) & =-1, \lambda(b)=1, \\
\sigma: D_{8} \rightarrow \mathbb{C}^{*}, \quad \sigma(a) & =1, \quad \sigma(b)=-1, \\
\nu: D_{8} \rightarrow U(2), \quad \nu(a) & =\left(\begin{array}{cc}
i & 0 \\
0 & -i
\end{array}\right), \quad \nu(b)=\left(\begin{array}{ll}
0 & 1 \\
1 & 0
\end{array}\right) .
\end{aligned}
$$

Therefore we have that $R\left(D_{8}\right)=\mathbb{Z}\langle\mathbf{1}, \lambda, \sigma, \sigma \otimes \lambda, \nu\rangle$, where the ring structure is given by the relations $\sigma \otimes \sigma=\mathbf{1}=\lambda \otimes \lambda$ and $\lambda \otimes \nu=\nu=\sigma \otimes \nu$.

6.1. $G=D_{8}$ and $A=\mathbb{Z} / 4$. Let us apply Theorem 3.2 to $K_{D_{8}}^{*}(*)=R\left(D_{8}\right)$ whenever $A=\mathbb{Z} / 4=\langle a\rangle$. In this case $Q=\mathbb{Z} / 2$ and the set orbits of the $D_{8}$ action on $\operatorname{Irr}(\mathbb{Z} / 4)=\left\{\mathbf{1}, \rho, \rho^{2}, \rho^{3}\right\}$ is $D_{8} \backslash \operatorname{Irr}(\mathbb{Z} / 4)=\left\{\{\mathbf{1}\},\left\{\rho, \rho^{3}\right\},\left\{\rho^{2}\right\}\right\}$ with $G_{[\rho]}=\mathbb{Z} / 4$; therefore we have the isomorphism

$$
K_{D_{8}}^{*}(*) \cong K_{D_{8}, 1}^{*}(*) \oplus K_{\mathbb{Z} / 4, \rho}^{*}(*) \oplus K_{D_{8}, \rho^{2}}^{*}(*) .
$$

Now, $K_{D_{8}, 1}^{*}(*)$ consists of the representations of $D_{8}$ that trivialize once restricted to $\langle a\rangle=\mathbb{Z} / 4$ and therefore

$$
K_{D_{8}, \mathbf{1}}^{*}(*)=\mathbb{Z}\langle\mathbf{1}, \sigma\rangle,
$$

the group $K_{D_{8}, \rho^{2}}^{*}(*)$ consists of the representations of $D_{8}$ that restrict to $\rho^{2}$, hence

$$
K_{D_{8}, \rho^{2}}^{*}(*)=\mathbb{Z}\langle\lambda, \sigma \otimes \lambda\rangle,
$$

and the group $K_{\mathbb{Z} / 4, \rho}^{*}(*)$ is by definition $\mathbb{Z}\langle\rho\rangle$. Recall from the proof of Theorem 3.2 that we can construct a $D_{8}$ representation out of an element $\rho \in K_{\mathbb{Z} / 4, \rho}^{*}(*)$. The construction is as follows. Take the representation $\rho$ which acts in the 1-dimensional vector space $V=\mathbb{C}$ by the homomorphism $\rho: \mathbb{Z} / 4 \rightarrow \mathbb{C}^{*}, 1 \mapsto i$, take the elements 
$\{1, b\}$ as the representatives of the orbits $G / G_{[\rho]}=\mathbb{Z} / 2$ and define the vector space $V \oplus V$ where the action of $D_{8}$ on $V \oplus V$ is generated by the equations

$$
\begin{gathered}
a \circ\left(v_{b}, v_{1}\right):=\left(\rho\left(a^{3}\right) v_{b} \oplus \rho(a) v_{1}\right)=\left(-i v_{b}, i v_{1}\right) \\
b \circ\left(v_{b}, v_{1}\right):=\left(v_{b b}, v_{b}\right)=\left(v_{1}, v_{b}\right) .
\end{gathered}
$$

The $D_{8}$ action on $V \oplus V$ gives precisely the irreducible representation $\nu$ and therefore the projection map $K_{D_{8}}^{*}(*) \rightarrow K_{\mathbb{Z} / 4, \rho}^{*}(*)$ projects $\nu \mapsto \rho$.

Now let us calculate $K_{D_{8}}^{*}(E \mathbb{Z} / 2)$ using the decomposition theorem:

$$
K_{D_{8}}^{*}(E \mathbb{Z} / 2)=K_{D_{8}, \mathbf{1}}^{*}(E \mathbb{Z} / 2) \oplus K_{\mathbb{Z} / 4, \rho}^{*}(E \mathbb{Z} / 2) \oplus K_{D_{8}, \rho^{2}}^{*}(E \mathbb{Z} / 2) .
$$

The first component $K_{D_{8}, \mathbf{1}}^{*}(E \mathbb{Z} / 2)$ becomes $K^{*}(B \mathbb{Z} / 2)$ since the subgroup $A=$ $\mathbb{Z} / 4$ must act trivially on the fibers. The second component $K_{\mathbb{Z} / 4, \rho}^{*}(E \mathbb{Z} / 2)$ becomes $\mathbb{Z}\langle\rho\rangle$ since $\mathbb{Z} / 4$ acts trivially on $E \mathbb{Z} / 2$ and $E \mathbb{Z} / 2$ is contractible. And the third component $K_{D_{8}, \rho^{2}}^{*}(E \mathbb{Z} / 2)$ becomes $K_{\mathbb{Z} / 2 \times \mathbb{Z} / 2, \beta}^{*}(E \mathbb{Z} / 2)$ with $\beta$ the non trivial irreducible representation of the left $\mathbb{Z} / 2$ since the representation $\rho^{2}$ is trivial on $a^{2}$; moreover the group $K_{\mathbb{Z} / 2 \times \mathbb{Z} / 2, \beta}^{*}(E \mathbb{Z} / 2)$ is isomorphic to $K^{*}(B \mathbb{Z} / 2)$ since $K_{\mathbb{Z} / 2, \beta}^{*}(*)=\mathbb{Z}\langle\beta\rangle$ and therefore we may apply the Kunneth isomorphism theorem for torsion free groups. Therefore we have the isomorphism

$$
K_{D_{8}}^{*}(E \mathbb{Z} / 2)=K^{*}(B \mathbb{Z} / 2) \oplus \mathbb{Z}\langle\rho\rangle \oplus K^{*}(B \mathbb{Z} / 2) .
$$

6.2. $G=D_{8}$ and $A=\mathbb{Z} / 2$. In the case that $A=Z\left(D_{8}\right)=\left\langle a^{2}\right\rangle=\mathbb{Z} / 2$ we have that $D_{8} \backslash \operatorname{Irr}(\mathbb{Z} / 2)=\{\{\mathbf{1}\},\{\rho\}\}$ where $\rho$ is the non trivial irreducible representation of $\mathbb{Z} / 2$, and $Q=D_{8} / Z\left(D_{8}\right)=(\mathbb{Z} / 2)^{2}$. In this case Theorem 3.2 becomes

$$
K_{D_{8}}^{*}(*)=K_{D_{8}, 1}^{*}(*) \oplus K_{D_{8}, \rho}^{*}(*)
$$

with $K_{D_{8}, \mathbf{1}}^{*}(*)=\mathbb{Z}\langle\mathbf{1}, \sigma, \lambda, \sigma \otimes \lambda\rangle \cong K_{(\mathbb{Z} / 2)^{2}}^{*}(*)$ and $K_{D_{8}, \rho}(*)=\langle\nu\rangle$.

Let us now see what happens if we apply the completion theorem component by component. The kernel of the restriction map $R\left(D_{8}\right) \rightarrow R(\mathbb{Z} / 2)$ is the ideal $I(Q)$ generated by the elements $\{\mathbf{1}-\lambda, \mathbf{1}-\sigma, \mathbf{1}-\sigma \otimes \lambda\}$, and since the action of this ideal on the representation $\nu$ is trivial, we conclude that $\Psi_{*}^{\rho}\left(I_{Q}^{n} \cdot K_{D_{8}}^{*}(*)\right)=0$ and therefore $K_{D_{8}, \rho}^{*}(*)=\widehat{K_{D_{8}, \rho}^{*}}(*)_{I_{Q}}$. Hence we obtain the following isomorphisms

$$
\begin{aligned}
K_{D_{8}}^{*}\left(E(\mathbb{Z} / 2)^{2}\right) & \left.\cong \widehat{K_{D_{8}}^{*}(*)}\right)_{I_{Q}} \cong \widehat{K_{D_{8}, \rho}^{*}}(*)_{I_{Q}} \oplus \widehat{K_{D_{8}, 1}^{*}}(*)_{I_{Q}} \\
& \cong{K_{D_{8}, \rho}^{*}(*) \oplus \widehat{K_{Q}^{*}(*)}}_{I_{Q}} \cong\langle\nu\rangle \oplus K^{*}\left(B(\mathbb{Z} / 2)^{2}\right) .
\end{aligned}
$$

Therefore $K_{D_{8}}^{*}\left(E(\mathbb{Z} / 2)^{2}\right)$ is the direct sum of the K-theory of $B(\mathbb{Z} / 2)^{2}$ and the free group generated by the $D_{8}$-equivariant vector bundle $\nu \times E D_{8} \rightarrow E D_{8}$.

The Atiyah-Hirzebruch-Segal spectral sequence associated to $K_{D_{8}}^{*}\left(E(\mathbb{Z} / 2)^{2}\right)$ has a second page isomorphic to Bredon cohomology $H_{D_{8}}^{*}\left(E(\mathbb{Z} / 2)^{2} ; R(-)\right)$ which is isomorphic to the ring $H^{*}\left(B(\mathbb{Z} / 2)^{2}, \mathbb{Z}\right) \otimes_{\mathbb{Z}} R(\mathbb{Z} / 2)$ since the isotropy is constant and $D_{8}$ acts trivially on $R(\mathbb{Z} / 2)$. This ring is isomorphic to the ring

$$
\mathbb{Z}\left[x^{2}, y^{2}, x^{2} y+x y^{2}, \alpha\right] /\left(\alpha^{2}-1,2 x^{2}, 2 y^{2}\right)
$$

where $|\alpha|=0$ and $|x|=|y|=1$; here we have used the fact that $H^{*}\left(B(\mathbb{Z} / 2)^{2}, \mathbb{Z}\right)$ is the kernel of the Steenrod square operation $S q^{1}$ in the $\operatorname{ring} H^{*}\left(B(\mathbb{Z} / 2)^{2}, \mathbb{F}_{2}\right)=$ $\mathbb{F}_{2}[x, y]$. 
The differential $d_{3}$ is defined on generators as $d_{3}(\alpha)=\left(x^{2} y+x y^{2}\right) \alpha$ and $d_{3} p(x, y)=$ $S q_{\mathbb{Z}}^{3}(p(x, y))$ on any polynomial on $x$ and $y$. Since the differential preserves the $R(\mathbb{Z} / 2)$ structure we have that the fourth page is a direct sum

$$
H^{*}\left(H^{*}\left(B(\mathbb{Z} / 2)^{2}, \mathbb{Z}\right), S q_{\mathbb{Z}}^{3}\right) \oplus H^{*}\left(H^{*}\left(B(\mathbb{Z} / 2)^{2}, \mathbb{Z}\right), S q_{\mathbb{Z}}^{3}+\left(x^{2} y+x y^{2}\right) \cup\right) .
$$

The left hand side was calculated by Atiyah [3, pp. 285] and the cohomology becomes

$$
\mathbb{Z}\left[x^{2}, y^{2}\right] /\left(x^{4} y^{2}-x^{2} y^{4}, 2 x^{2}, 2 y^{2}\right)
$$

and since everything is of even degree, the spectral sequence collapses at the fourth page. The cohomology of the right hand side is localized in degree 0 and is isomorphic to $\mathbb{Z} \cong \mathbb{Z}\langle 2 \alpha\rangle$ (one can check that the differential $S q^{3}+\left(x^{2} y+x y^{2}\right) \cup$ on $\mathbb{F}_{2}\left[x^{2}, y^{2}, x^{2} y+x y^{2}\right]$ has trivial cohomology).

Therefore the page at infinity becomes

$$
E_{\infty}=\mathbb{Z}\left[x^{2}, y^{2}\right] /\left(x^{4} y^{2}-x^{2} y^{4}, 2 x^{2}, 2 y^{2}\right) \oplus \mathbb{Z}\langle 2 \alpha\rangle
$$

where the ring $\mathbb{Z}\left[x^{2}, y^{2}\right] /\left(x^{4} y^{2}-x^{2} y^{4}, 2 x^{2}, 2 y^{2}\right)$ corresponds to the associated graded of $K^{*}\left(B(\mathbb{Z} / 2)^{2}\right)$ and $\mathbb{Z}\langle 2 \alpha\rangle$ corresponds to $K_{D_{8}, \rho}^{*}(*)=\mathbb{Z}\langle\nu\rangle$ with $\nu \mapsto 2 \alpha$.

In particular note that the image of edge homomorphism of the spectral sequence

$$
K_{D^{8}}^{0}\left(E(\mathbb{Z} / 2)^{2}\right) \rightarrow H^{0}\left(B(\mathbb{Z} / 2)^{2}, \mathbb{Z}\right) \otimes_{\mathbb{Z}} R(\mathbb{Z} / 2) \cong R(\mathbb{Z} / 2)
$$

is not sujective. The image is $\mathbb{Z}\langle 1,2 \alpha\rangle \subset \mathbb{Z}\langle 1, \alpha\rangle=R(\mathbb{Z} / 2)$.

\section{REFERENCES}

[1] A. Adem and R. J. Milgram. Cohomology of Finite Groups. Number 309 in Grundlehren der mathematischen Wissenschaften. Springer-Verlag Berlin Heidelberg, second edition, 2004.

[2] A. Adem and Y. Ruan. Twisted orbifold K-theory. Comm. Math. Phys., 237(3):533-556, 2003.

[3] M. F. Atiyah. Characters and cohomology of finite groups. Inst. Hautes Études Sci. Publ. Math., (9):23-64, 1961.

[4] M. F. Atiyah and G. Segal. Equivariant $K$-theory and completion. J. Differential Geometry, $3: 1-18,1969$.

[5] M. F. Atiyah and G. Segal. Twisted K-theory. Ukr. Mat. Visn., 1(3):287-330, 2004.

[6] M. F. Atiyah and G. Segal. Twisted K-theory and cohomology. In Inspired by S. S. Chern, volume 11 of Nankai Tracts Math., pages 5-43. World Sci. Publ., Hackensack, NJ, 2006.

[7] K. S. Brown. Cohomology of groups. Number 309 in Graduate Texts in Mathemat- ics. Springer-Verlag, New York, second edition, 1994.

[8] S. Jackowski. Families of subgroups and completion. J. Pure Appl. Algebra, 37(2):167-179, 1985.

[9] G. Segal. Classifying spaces and spectral sequences. Inst. Hautes Études Sci. Publ. Math., (34):105-112, 1968.

[10] G. Segal. Equivariant K-theory. Inst. Hautes Études Sci. Publ. Math., (34):129-151, 1968.

Escuela de Matemáticas, Universidad Nacional de Colombia sede Medellín, Calle 59A No 63-20, Bloque 43, Oficina 243, Medellín, Colombia

E-mail address: jmgomez0@unal.edu.co

$U R L:$ https://sites.google.com/a/unal.edu.co/jmgomez0/

Departamento de Matemáticas y Estadística, Universidad del Norte, Km. 5 via Puerto Colombia, Barranquilla, Colombia

E-mail address: bjongbloed@uninorte.edu.co

$U R L:$ https://sites.google.com/site/bernardouribejongbloed/ 